\author{
ANTONOVA T.M. ${ }^{1}$, DMYTRYSHYN M.V. ${ }^{2}$, VOZNA S.M. ${ }^{1}$
}

\title{
SOME PROPERTIES OF APPROXIMANTS FOR BRANCHED CONTINUED FRACTIONS OF THE SPECIAL FORM WITH POSITIVE AND ALTERNATING-SIGN PARTIAL NUMERATORS
}

\begin{abstract}
The paper deals with research of convergence for one of the generalizations of continued fractions - branched continued fractions of the special form with two branches. Such branched continued fractions, similarly as the two-dimensional continued fractions and the branched continued fractions with two independent variables are connected with the problem of the correspondence between a formal double power series and a sequence of the rational approximants of a function of two variables.

Unlike continued fractions, approximants of which are constructed unambiguously, there are many ways to construct approximants of branched continued fractions of the general and the special form. The paper examines the ordinary approximants and one of the structures of figured approximants of the studied branched continued fractions, which is connected with the problem of correspondence.

We consider some properties of approximants of such fractions, whose partial numerators are positive and alternating-sign and partial denominators are equal to one. Some necessary and sufficient conditions for figured convergence are established. It is proved that under these conditions from the convergence of the sequence of figured approximants it follows the convergence of the sequence of ordinary approximants to the same limit.

Key words and phrases: branched continued fraction of the special form, ordinary approximants, figured approximants, convergence, figured convergence.
\end{abstract}

${ }^{1}$ Lviv Polytechnic National University, 12 Bandera str., 79013, Lviv, Ukraine

2 Ternopil National Economic University, 11 Lvivska str., 46020, Ternopil, Ukraine

E-mail: tamara_antonova@ukr.net (Antonova T.M.), martadmytryshyn@hotmail . com (Dmytryshyn M.V.), svitlanavozna@gmail.com(Vozna S.M.)

\section{INTRODUCTION}

The paper is devoted to study of the branched continued fractions (BCF) of the form

$$
b_{0}+F_{0,0}+D_{i=1}^{\infty} \frac{a_{i, 0}}{1+F_{i, 0}}+D_{i=1}^{\infty} \frac{a_{0, i}}{1+F_{0, i}}
$$

where $F_{i, j}$ are continued fractions $(\mathrm{CF})$

$$
F_{i, j}=D_{p=1}^{\infty} \frac{a_{p+i, p+j}}{1}=1+\frac{a_{1+i, 1+j}}{1+\frac{a_{2+i, 2+j}}{1+} \cdot}, i=0,1, \ldots, \quad j=0,1, \ldots
$$

$\mathrm{y} \Delta \mathrm{K} 517.524$

2010 Mathematics Subject Classification: 11A55, 11J70, 30B70, 40A15. 
$b_{0}, a_{k, j}, j=0,1, \ldots, k=0,1, \ldots, k+j \geq 1$, are complex numbers or functions of two variables which are defined in some set $D \subset \mathbb{C}^{2}$.

If all elements of BCF (1)-(2) are numbers, then BCF (1)-(2) is said to be numerical BCF of the special form. If some or all elements of BCF (1)-(2) are functions, then BCF (1)-(2) is said to be functional BCF of the special form.

The above mentioned BCF is one of two-dimensional generalizations of continued fractions, which were offered for the solution of correspondence problem between a formal double power series (FDPS) and a sequence of the rational approximants of a function of two variables $[9,12,13]$. Functional BCF of the special form is corresponding to FDPS

$$
\sum_{i+j \geq 0}^{\infty} c_{i, j} z_{1}^{i} z_{2}^{j}
$$

if the expansion of its $n$th approximant into FDPS $\sum_{i+j \geq 0}^{\infty} c_{i, j}^{(n)} z_{1}^{i} z_{2}^{j}$ coincides with the FDPS (3) to all terms of power $n$ inclusively, that is $c_{i, j}^{(n)}=c_{i, j}, i+j \leq n$.

In the paper [13] it is shown, that BCF (1)-(2) is corresponding to the FDPS (3), if $b_{0}=c_{0,0}, a_{i, 0}=b_{i, 0} z_{1}, a_{0, i}=b_{0, i} z_{2}, a_{i, j}=b_{i, j} z_{1} z_{2}, i, j=1,2, \ldots$, the coefficients $b_{k, 0}, b_{0, k}, a_{k, j}$, $j, k=1,2, \ldots$, are calculating by formulas in term of the coefficients of the FDPS (3), and the $n$th approximants $\tilde{f}_{n}$ are defined as follows

$$
\tilde{f}_{0}=b_{0}, \tilde{f}_{n}=b_{0}+F_{0,0}^{\left(\left[\frac{n}{2}\right]\right)}+D_{k=1}^{n} \frac{a_{i, 0}}{1+F_{i, 0}^{\left(\left[\frac{n-i}{2}\right]\right)}}+D_{k=1}^{n} \frac{a_{0, i}}{1+F_{0, i}^{\left(\left[\frac{n-i}{2}\right]\right)}}, n=1,2, \ldots,
$$

where $[\alpha]$ is an integer part of a real number $\alpha$,

$$
F_{i, j}^{(0)}=0, \quad F_{i, j}^{(k)}=D_{p=1}^{k} \frac{a_{p+i, p+j}}{1}, \quad i, j=0,1, \ldots, \quad k=1,2, \ldots
$$

Finite continued fractions (5) are called the $k$ th approximants of CF (2).

We can construct the approximants of BCF in different ways. Ordinary $n$th approximants of BCF (1)-(2) are defined as follows

$$
f_{0}=b_{0}, f_{n}=b_{0}+F_{0,0}^{(n)}+D_{k=1}^{n} \frac{a_{i, 0}}{1+F_{i, 0}^{(n-i)}}+D_{k=1}^{n} \frac{a_{0, i}}{1+F_{0, i}^{(n-i)}}, n=1,2, \ldots
$$

Approximants $\tilde{f}_{n}$ from (4) are examples of so called figured approximants [5]. Expressions

$$
\begin{aligned}
& Q_{i, 0}^{(0)}=1, Q_{i, 0}^{(k+1)}=1+F_{i, 0}^{(k+1)}+\frac{a_{i+1,0}}{Q_{i+1,0}^{(k)}}, \quad i=1,2, \ldots, \quad k=0,1, \ldots, \\
& Q_{0, i}^{(0)}=1, Q_{0, i}^{(k+1)}=1+F_{0, i}^{(k+1)}+\frac{a_{0, i+1}}{Q_{0, i+1}^{(k)}}, \quad i=1,2, \ldots, \quad k=0,1, \ldots,
\end{aligned}
$$

are said to be the tails of ordinary approximants for BCF (1)-(2). Tails of figured approximants (4) for BCF (1)-(2) are defined by following formulas

$$
\tilde{Q}_{i, 0}^{(0)}=1, \tilde{Q}_{i, 0}^{(k+1)}=1+F_{i, 0}^{\left(\left[\frac{k+1}{2}\right]\right)}+\frac{a_{i+1,0}}{\tilde{Q}_{i+1,0}^{(k)}}, \quad i=1,2, \ldots, \quad k=0,1, \ldots,
$$




$$
\tilde{Q}_{0, i}^{(0)}=1, \tilde{Q}_{0, i}^{(k+1)}=1+F_{0, i}^{\left(\left[\frac{k+1}{2}\right]\right)}+\frac{a_{0, i+1}}{\tilde{Q}_{0, i+1}^{(k)}}, \quad i=1,2, \ldots, \quad k=0,1, \ldots
$$

Taking into account notations (6)-(9), it is possible to write

$$
\begin{gathered}
f_{n}=b_{0}+F_{0,0}^{(n)}+\frac{a_{1,0}}{Q_{1,0}^{(n-1)}}+\frac{a_{0,1}}{Q_{0,1}^{(n-1)}}, n=1,2, \ldots \\
\tilde{f}_{n}=b_{0}+F_{0,0}^{\left(\left[\frac{n}{2}\right]\right)}+\frac{a_{1,0}}{\tilde{Q}_{1,0}^{(n-1)}}+\frac{a_{0,1}}{\tilde{Q}_{0,1}^{(n-1)}}, n=1,2, \ldots
\end{gathered}
$$

Approximants $f_{k}, \tilde{f}_{k}$ have sense if in process of reduction of BCF (calculations of their tails by formulas (6)-(9)) uncertainty of the type $\frac{0}{0}$ don't appears (it is assumed, that $\frac{1}{0}=\infty$, $\frac{1}{\infty}=0$ and $\frac{\alpha_{1}}{0}+\ldots+\frac{\alpha_{m}}{0}=\frac{0}{0}$, if $\left.m>1\right)$. We say that BCF (1)-(2) is figured convergent if, beginning from some number $n_{0}$, all its figured approximants have sense and there is finite limit $\tilde{f}=\lim _{n \rightarrow \infty} \tilde{f}_{n}$. The value of this limit can be the value of figured convergent BCF.

BCF (1)-(2) is said to be convergent, if beginning from some number $n_{0}$, all its ordinary approximants have sense and there is finite limit $f=\lim _{n \rightarrow \infty} f_{n}$. The value of this limit also it is possible to assume as the value of convergent $\mathrm{BCF}$.

BCF (1)-(2) is said to be divergent (figured divergent) if infinite numbers of its approximants (figured approximants) have not sense or there isn't only one finite limit of sequence of its approximants (figured approximants).

A lot of works of analytic theory of multidimensional generalization for continued fractions are devoted to research of convergence $[5,10]$. This problem is important till now $[3,4,6,7]$.

For research of properties of sequences of approximants for BCF of the special form the formulas of difference for two their approximants are used. There are such formulas [5]:

$$
\begin{gathered}
\tilde{f}_{n}-\tilde{f}_{m}=F_{0,0}^{\left(\left[\frac{n}{2}\right]\right)}-F_{0,0}^{\left(\left[\frac{m}{2}\right]\right)}+\sum_{i=1}^{m} \frac{(-1)^{i}\left(F_{i, 0}^{\left(\left[\frac{n-i}{2}\right]\right)}-F_{i, 0}^{\left(\left[\frac{m-i}{2}\right]\right)}\right) \prod_{j=1}^{i} a_{j, 0}}{\prod_{j=1}^{i} \tilde{Q}_{j, 0}^{(n-j)} \tilde{Q}_{j, 0}^{(m-j)}}+\frac{(-1)^{m} \prod_{j=1}^{m+1} a_{j, 0}}{\prod_{j=1}^{m+1} \tilde{Q}_{j, 0}^{(n-j)} \prod_{j=1}^{m} \tilde{Q}_{j, 0}^{(m-j)}} \\
+\sum_{i=1}^{m} \frac{(-1)^{i}\left(F_{0, i}^{\left(\left[\frac{n-i}{2}\right]\right)}-F_{0, i}^{\left(\left[\frac{m-i}{2}\right]\right)}\right) \prod_{j=1}^{i} a_{0, j}}{\prod_{j=1}^{i} \tilde{Q}_{0, j}^{(n-j)} \tilde{Q}_{0, j}^{(m-j)}}+\frac{(-1)^{m} \prod_{j=1}^{m+1} a_{0, j}}{\prod_{j=1}^{m+1} \tilde{Q}_{0, j}^{(n-j)} \prod_{j=1}^{m} \tilde{Q}_{0, j}^{(m-j)}}, n>m, \text { and } \\
f_{n}-\tilde{f}_{m}=F_{0,0}^{(n)}-F_{0,0}^{\left(\left[\frac{m}{2}\right]\right)}+\sum_{i=1}^{m} \frac{(-1)^{i}\left(F_{i, 0}^{(n-i)}-F_{i, 0}^{\left(\left[\frac{m-i}{2}\right]\right)}\right) \prod_{j=1}^{i} a_{j, 0}}{\prod_{j=1}^{i} Q_{j, 0}^{(n-j)} \tilde{Q}_{j, 0}^{(m-j)}} \frac{(-1)^{m} \prod_{j=1}^{m+1} a_{j, 0}}{\prod_{j=1}^{m+1} Q_{j, 0}^{(n-j)} \prod_{j=1}^{m} \tilde{Q}_{j, 0}^{(m-j)}} \\
+\sum_{i=1}^{m} \frac{(-1)^{i}\left(F_{0, i}^{(n-i)}-F_{0, i}^{\left(\left[\frac{m-i}{2}\right]\right)}\right) \prod_{j=1}^{i} a_{0, j}}{\prod_{j=1}^{i} Q_{0, j}^{(n-j)} \tilde{Q}_{0, j}^{(m-j)}}+\frac{(-1)^{m} \prod_{j=1}^{m+1} a_{0, j}}{\prod_{j=1}^{m+1} Q_{0, j}^{(n-j)} \prod_{j=1}^{m} \tilde{Q}_{0, j}^{(m-j)}}, n>m .
\end{gathered}
$$

We note that the formulas (10)-(11) have been established in assumption, that the values of all tails $\tilde{Q}_{0, k}^{(p)}, \tilde{Q}_{k, 0}^{(p)}, Q_{0, k}^{(p)}, Q_{k, 0}^{(p)}$, which appear in these formulas, differ from 0 . 


\section{MAIN RESULTS}

This work is the continuation of the study of properties of approximants for numerical BCF (1)-(2) with real elements [1,2]. We will consider BCF which elements satisfy such conditions

$$
a_{i, j}>0,\left|a_{i, 0}\right|=(-1)^{i-1} a_{i, 0} \neq 0,\left|a_{0, i}\right|=(-1)^{i-1} a_{0, i} \neq 0, i, j=1,2, \ldots .
$$

Under the conditions (12) $F_{0,0}, F_{i, 0}, F_{0, i}$ are the continued fractions with positive elements. It is well known $[8,11]$ that approximants of even order for such fractions generate a monotone increasing sequence, approximants of odd order for such fractions generate a monotone decreasing sequence and all approximants of even order are less than every approximants of odd order. Taking these results into account, we have

$$
\begin{aligned}
& F_{0,0}^{(2 m)}<F_{0,0}^{(2 m+2)}<F_{0,0}^{(2 n+1)}<F_{0,0}^{(2 n-1)}, \quad m=0,1, \ldots, \quad n=1,2, \ldots, \\
& F_{i, 0}^{(2 m)}<F_{i, 0}^{(2 m+2)}<F_{i, 0}^{(2 n+1)}<F_{i, 0}^{(2 n-1)}, \quad m=0,1, \ldots, \quad n=1,2, \ldots, \\
& F_{0, i}^{(2 m)}<F_{0, i}^{(2 m+2)}<F_{0, i}^{(2 n+1)}<F_{0, i}^{(2 n-1)}, \quad m=0,1, \ldots, \quad n=1,2, \ldots .
\end{aligned}
$$

Theorem 1. Let the elements of BCF (1)-(2) satisfy the conditions (12) and

$$
1+a_{2 i, 0}>0, \quad 1+a_{0,2 i}>0, \quad i=1,2, \ldots
$$

Then the following inequalities are true

$$
\begin{gathered}
\tilde{f}_{4 m}<\tilde{f}_{4 m+4}<\tilde{f}_{4 n+2}<\tilde{f}_{4 n-2}, \quad m=0,1, \ldots, \quad n=1,2, \ldots, \\
\tilde{f}_{4 m}<\tilde{f}_{4 m+2 p+3}<\tilde{f}_{4 m+2}, \quad m=0,1, \ldots, \quad p=1,2, \ldots,
\end{gathered}
$$

and the sequences $\left\{\tilde{f}_{4 p}\right\},\left\{\tilde{f}_{4 p+2}\right\}, p=0,1, \ldots$, converge.

Proof. Let $k$ be an arbitrary natural number. Using definitions (8)-(9), by induction on $p$ let us show that the following inequalities are valid

$$
\begin{gathered}
1 \leq \tilde{Q}_{2 k, 0}^{(p)} \leq 1+a_{2 k+1,1}+\frac{a_{2 k+1,0}}{1-\left|a_{2 k+2,0}\right|}, \quad p=0,1, \ldots, \\
1-\left|a_{2 k, 0}\right| \leq \tilde{Q}_{2 k-1,0}^{(p)} \leq 1+a_{2 k, 1}, \quad p=0,1, \ldots .
\end{gathered}
$$

Indeed, for $p=0$ and $p=1$ we have

$$
\tilde{Q}_{2 k-1,0}^{(0)}=\tilde{Q}_{2 k, 0}^{(0)}=1, \quad 0<1-\left|a_{2 k, 0}\right|=\tilde{Q}_{2 k-1,0}^{(1)}<1, \quad \tilde{Q}_{2 k, 0}^{(1)}=1+a_{2 k+1,0}, \quad k=1,2, \ldots
$$

In assumption that inequality (19) is true for $p=r$, we obtain

$$
0<1-\left|a_{2 k, 0}\right|<1+F_{2 k-1,0}^{\left(\left[\frac{p+1}{2}\right]\right)}-\frac{\left|a_{2 k, 0}\right|}{1} \leq \tilde{Q}_{2 k-1,0}^{(p+1)}=1+F_{2 k-1,0}^{\left(\left[\frac{p+1}{2}\right]\right)}-\frac{\left|a_{2 k, 0}\right|}{\tilde{Q}_{2 k, 0}^{(p)}}<1+a_{2 k, 1},
$$

i.e. (20) is valid for $p=r+1$. Assuming that inequality (20) holds true for $p=r$ we get

$$
1<1+\frac{a_{2 k+1,0}}{1+a_{2 k+2,1}}<\tilde{Q}_{2 k, 0}^{(p+1)}=1+F_{2 k, 0}^{\left(\left[\frac{p+1}{2}\right)\right.}+\frac{a_{2 k+1,0}}{\tilde{Q}_{2 k+1,0}^{(p)}} \leq 1+a_{2 k+1,1}+\frac{a_{2 k+1,0}}{1-\left|a_{2 k+2,0}\right|}
$$


i.e. inequality (19) is valid for $p=r+1$. Hence, estimations (19)-(20) are true for arbitrary $k, p$. Analogously we verify validity of inequalities

$$
\begin{gathered}
1 \leq \tilde{Q}_{0,2 k}^{(p)} \leq 1+a_{1,2 k+1}+\frac{a_{0,2 k+1}}{1-\left|a_{0,2 k+2}\right|}, \quad k=1,2, \ldots, \quad p=0,1, \ldots, \\
1-\left|a_{0,2 k}\right| \leq \tilde{Q}_{0,2 k-1}^{(p)} \leq 1+a_{1,2 k}, \quad k=1,2, \ldots, \quad p=0,1, \ldots .
\end{gathered}
$$

Further we consider the differences $\tilde{f}_{4 m+2 p+l}-\tilde{f}_{4 m}, \tilde{f}_{4 n+2 p+l-2}-\tilde{f}_{4 n-2}, m, n, p=1,2, \ldots$, $l=0,1, \ldots$, using the formula (10). Let

$$
\begin{aligned}
& \tilde{Z}_{n, 0}^{(1)}=0, \quad \tilde{Z}_{n, m}^{(1)}=\sum_{i=1}^{m} \frac{(-1)^{i}\left(F_{i, 0}^{\left(\left[\frac{n-i}{2}\right]\right)}-F_{i, 0}^{\left(\left[\frac{m-i}{2}\right]\right)}\right) \prod_{j=1}^{i} a_{j, 0}}{\prod_{j=1}^{i} \tilde{Q}_{j, 0}^{(n-j)} \tilde{Q}_{j, 0}^{(m-j)}}, n>m ; \\
& \tilde{Z}_{n, 0}^{(2)}=0, \quad \tilde{Z}_{n, m}^{(2)}=\sum_{i=1}^{m} \frac{(-1)^{i}\left(F_{0, i}^{\left(\left[\frac{n-i}{2}\right]\right)}-F_{0, i}^{\left(\left[\frac{m-i}{2}\right]\right)}\right) \prod_{j=1}^{i} a_{0, j}}{\prod_{j=1}^{i} \tilde{Q}_{0, j}^{(n-j)} \tilde{Q}_{0, j}^{(m-j)}}, n>m .
\end{aligned}
$$

Then

$$
\begin{aligned}
\tilde{f}_{4 m+2 p+l}-\tilde{f}_{4 m} & =F_{0,0}^{\left(2 m+p+\left[\frac{l}{2}\right]\right)}-F_{0,0}^{(2 m)}+\tilde{Z}_{4 m+2 p+l, 4 m}^{(1)}+\tilde{Z}_{4 m+2 p+l, 4 m}^{(2)} \\
& +\frac{\prod_{j=1}^{4 m+1} a_{j, 0}}{\prod_{j=1}^{4 m+1} \tilde{Q}_{j, 0}^{(4 m+2 p+l-j)} \prod_{j=1}^{4 m} \tilde{Q}_{j, 0}^{(4 m-j)}}+\frac{\prod_{j=1}^{4 m+1} a_{0, j}}{\prod_{j=1}^{4 m+1} \tilde{Q}_{0, j}^{(4 m+2 p+l-j)} \prod_{j=1}^{4 m} \tilde{Q}_{0, j}^{(4 m-j)}}
\end{aligned}
$$

Taking into account conditions (12) and inequalities (13)-(15), (19)-(22), we have

$$
\begin{gathered}
\prod_{j=1}^{4 m+1} a_{j, 0}=\prod_{j=1}^{4 m+1}\left|a_{j, 0}\right|>0, \prod_{j=1}^{4 m+1} a_{0, j}=\prod_{j=1}^{4 m+1}\left|a_{0, j}\right|>0, \\
\tilde{Z}_{4 m+2 p+l, 4 m}^{(1)}=-\sum_{i=1}^{m} \frac{\left(F_{4 i-3,0}^{\left(2 m-2 i+p+1+\left[\frac{l+1}{2}\right]\right)}-F_{4 i-3,0}^{(2 m-2 i+1)}\right) \prod_{j=1}^{4 i-3}\left|a_{j, 0}\right|}{\prod_{j=1}^{4 i-3} \tilde{Q}_{j, 0}^{(4 m+2 p+l-j)} \tilde{Q}_{j, 0}^{(4 m-j)}} \\
-\sum_{i=1}^{m} \frac{\left(F_{4 i-2,0}^{\left(2 m-2 i+p+1+\left[\frac{l}{2}\right]\right)}-F_{4 i-2,0}^{(2 m-2 i+1)}\right) \prod_{j=1}^{4 i-2}\left|a_{j, 0}\right|}{\prod_{j=1}^{4 i-2} \tilde{Q}_{j, 0}^{(4 m+2 p+l-j)} \tilde{Q}_{j, 0}^{(4 m-j)}}+\sum_{i=1}^{m} \frac{\left(F_{4 i-1,0}^{\left(2 m-2 i+p+\left[\frac{l+1}{2}\right]\right)}-F_{4 i-1,0}^{(2 m-2 i)}\right) \prod_{j=1}^{4 i-1}\left|a_{j, 0}\right|}{\prod_{j=1}^{4 i-1} \tilde{Q}_{j, 0}^{(4 m+2 p+l-j)} \tilde{Q}_{j, 0}^{(4 m-j)}} \\
+\sum_{i=1}^{m} \frac{\left(F_{4 i, 0}^{\left(2 m-2 i+p+\left[\frac{l}{2}\right]\right)}-F_{4 i, 0}^{(2 m-2 i)}\right) \prod_{j=1}^{4 i}\left|a_{j, 0}\right|}{\prod_{j=1}^{4 i} \tilde{Q}_{j, 0}^{(4 m+2 p+l-j)} \tilde{Q}_{j, 0}^{(4 m-j)}}=\sum_{i=1}^{m} \frac{\left|F_{4 i-3,0}^{\left(2 m-2 i+p+1+\left[\frac{l+1}{2}\right]\right)}-F_{4 i-3,0}^{(2 m-2 i+1)}\right| \prod_{j=1}^{4 i-3}\left|a_{j, 0}\right|}{\prod_{j=1}^{4 i-3} \tilde{Q}_{j, 0}^{(4 m+2 p+l-j)} \tilde{Q}_{j, 0}^{(4 m-j)}}
\end{gathered}
$$




$$
\begin{aligned}
& +\sum_{i=1}^{m} \frac{\left|F_{4 i-2,0}^{\left(2 m-2 i+p+1+\left[\frac{l}{2}\right]\right)}-F_{4 i-2,0}^{(2 m-2 i+1)}\right| \prod_{j=1}^{4 i-2}\left|a_{j, 0}\right|}{\prod_{j=1}^{4 i-2} \tilde{Q}_{j, 0}^{(4 m+2 p+l-j)} \tilde{Q}_{j, 0}^{(4 m-j)}}+\sum_{i=1}^{m} \frac{\left|F_{4 i-1,0}^{\left(2 m-2 i+p+\left[\frac{l+1}{2}\right]\right)}-F_{4 i-1,0}^{(2 m-2 i)}\right| \prod_{j=1}^{4 i-1}\left|a_{j, 0}\right|}{\prod_{j=1}^{4 i-1} \tilde{Q}_{j, 0}^{(4 m+2 p+l-j)} \tilde{Q}_{j, 0}^{(4 m-j)}} \\
& +\sum_{i=1}^{m} \frac{\left|F_{4 i, 0}^{\left(2 m-2 i+p+\left[\frac{l}{2}\right]\right)}-F_{4 i, 0}^{(2 m-2 i)}\right| \prod_{j=1}^{4 i}\left|a_{j, 0}\right|}{\prod_{j=1}^{4 i} \tilde{Q}_{j, 0}^{(4 m+2 p+l-j)} \tilde{Q}_{j, 0}^{(4 m-j)}}>0 .
\end{aligned}
$$

Similarly $\tilde{Z}_{4 m+2 p+l, 4 m}^{(2)}>0$. Consequently

$$
\begin{aligned}
& \tilde{f}_{4 m+2 p+l}-\tilde{f}_{4 m}>0, \quad m, l=0,1, \ldots, \quad p=1,2, \ldots, \\
& \tilde{f}_{4 n+2 p+l-2}-\tilde{f}_{4 n-2}=F_{0,0}^{\left(2 n+p-1+\left[\frac{l}{2}\right]\right)}-F_{0,0}^{(2 n-1)}+\tilde{Z}_{4 n+2 p+l-2,4 n-2}^{(1)}+\tilde{Z}_{4 n+2 p+l-2,4 n-2}^{(2)} \\
& +\frac{\prod_{j=1}^{4 n-1} a_{j, 0}}{\prod_{j=1}^{4 n-1} \tilde{Q}_{j, 0}^{(4 n+2 p+l-2-j)} \prod_{j=1}^{4 n-2} \tilde{Q}_{j, 0}^{(4 n-2-j)}}+\frac{\prod_{j=1}^{4 n-1} a_{0, j}}{\prod_{j=1}^{4 n-1} \tilde{Q}_{0, j}^{(4 n+2 p+l-2-j)} \prod_{j=1}^{4 n-2} \tilde{Q}_{0, j}^{(4 n-2-j)}}, \\
& \prod_{j=1}^{4 n-1} a_{j, 0}=-\prod_{j=1}^{4 n-1}\left|a_{j, 0}\right|<0, \quad \prod_{j=1}^{4 n-1} a_{0, j}=-\prod_{j=1}^{4 n-1}\left|a_{0, j}\right|<0, \\
& \tilde{Z}_{4 n+2 p+l-2,4 n-2}^{(1)}=-\sum_{i=1}^{n} \frac{\left(F_{4 i-3,0}^{\left(2 n-2 i+p+\left[\frac{l+1}{2}\right]\right)}-F_{4 i-3,0}^{(2 n-2 i)}\right) \prod_{j=1}^{4 i-3}\left|a_{j, 0}\right|}{\prod_{j=1}^{4 i-3} \tilde{Q}_{j, 0}^{(4 n+2 p+l-2-j)} \tilde{Q}_{j, 0}^{(4 n-2-j)}} \\
& +\sum_{i=1}^{n-1} \frac{\left(F_{4 i-1,0}^{\left(2 n+p-2 i-1+\left[\frac{l+1}{2}\right]\right)}-F_{4 i-1,0}^{(2 n-2 i-1)}\right) \prod_{j=1}^{4 i-1}\left|a_{j, 0}\right|}{\prod_{j=1}^{4 i-1} \tilde{Q}_{j, 0}^{(4 n+2 p+l-2-j)} \tilde{Q}_{j, 0}^{(4 n-2-j)}}-\sum_{i=1}^{n} \frac{\left(F_{4 i-2,0}^{\left(2 n-2 i+p+\left[\frac{l}{2}\right]\right)}-F_{4 i-2,0}^{(2 n-2 i)}\right) \prod_{j=1}^{4 i-2}\left|a_{j, 0}\right|}{\prod_{j=1}^{4 i-2} \tilde{Q}_{j, 0}^{(4 n+2 p+l-2-j)} \tilde{Q}_{j, 0}^{(4 n-2-j)}} \\
& +\sum_{i=1}^{n-1} \frac{\left(F_{4 i, 0}^{\left(2 n+p-2 i-1+\left[\frac{l}{2}\right]\right)}-F_{4 i, 0}^{(2 n-2 i-1)}\right) \prod_{j=1}^{4 i}\left|a_{j, 0}\right|}{\prod_{j=1}^{4 i} \tilde{Q}_{j, 0}^{(4 n+2 p+l-2-j)} \tilde{Q}_{j, 0}^{(4 n-2-j)}}=-\sum_{i=1}^{n} \frac{\left|F_{4 i-3,0}^{\left(2 n-2 i+p+\left[\frac{l+1}{2}\right]\right)}-F_{4 i-3,0}^{(2 n-2 i)}\right| \prod_{j=1}^{4 i-3}\left|a_{j, 0}\right|}{\prod_{j=1}^{4 i-3} \tilde{Q}_{j, 0}^{(4 n+2 p+l-2-j)} \tilde{Q}_{j, 0}^{(4 n-j-2)}} \\
& -\sum_{i=1}^{n} \frac{\left|F_{4 i-2,0}^{\left(2 n-2 i+p+\left[\frac{l}{2}\right]\right)}-F_{4 i-2,0}^{(2 n-2 i)}\right| \prod_{j=1}^{4 i-2}\left|a_{j, 0}\right|}{\prod_{j=1}^{4 i-2} \tilde{Q}_{j, 0}^{(4 n+2 p+l-j-2)} \tilde{Q}_{j, 0}^{(4 n-j-2)}}-\sum_{i=1}^{n-1} \frac{\left|F_{4 i-1,0}^{\left(2 n-2 i+p-1+\left[\frac{l+1}{2}\right]\right)}-F_{4 i-1,0}^{(2 n-2 i-1)}\right| \prod_{j=1}^{4 i-1}\left|a_{j, 0}\right|}{\prod_{j=1}^{4 i-1} \tilde{Q}_{j, 0}^{(4 n+2 p+l-j-2)} \tilde{Q}_{j, 0}^{(4 n-j-2)}} \\
& -\sum_{i=1}^{n-1} \frac{\left|F_{4 i, 0}^{\left(2 n-2 i+p-1+\left[\frac{l}{2}\right]\right)}-F_{4 i, 0}^{(2 n-2 i-1)}\right| \prod_{j=1}^{4 i}\left|a_{j, 0}\right|}{\prod_{j=1}^{4 i} \tilde{Q}_{j, 0}^{(4 n+2 p+l-j-2)} \tilde{Q}_{j, 0}^{(4 n-j-2)}}<0, \quad \tilde{Z}_{4 n+2 p+l-2,4 n-2}^{(2)}<0 .
\end{aligned}
$$


Consequently

$$
\tilde{f}_{4 n+2 p+l-2}-\tilde{f}_{4 n-2}<0, \quad l=0,1, \ldots, \quad n, p=1,2, \ldots .
$$

From the inequalities (24), (26), where $l=0$, it follows "fork" property for figured approximants of even order. This property is described by system of inequalities (17). Therefore sequences $\left\{\tilde{f}_{4 k}\right\},\left\{\tilde{f}_{4 k+2}\right\}$ are monotone, bounded and convergent. From (24), (26), where $l=3, n=m+1$, we obtain inequality (18).

Proposition. BCF (1)-(2), with elements that satisfy conditions (12) and (16), is figured convergent if and only if $\lim _{n \rightarrow \infty}\left(\tilde{f}_{4 n+2}-\tilde{f}_{4 n}\right)=0$.

Proof. It is above mentioned that under conditions (12) and (16) the sequences $\left\{\tilde{f}_{4 k}\right\},\left\{\tilde{f}_{4 k+2}\right\}$ converge to finite limits. Condition $\lim _{n \rightarrow \infty}\left(\tilde{f}_{4 n+2}-\tilde{f}_{4 n}\right)=0$ implies equality of these limits, i.e. convergence of the sequence $\left\{\tilde{f}_{2 k}\right\}$. Taking into account inequality (18), we conclude that $\lim _{k \rightarrow \infty} \tilde{f}_{2 k}=\lim _{k \rightarrow \infty} \tilde{f}_{2 k-1}$, i.e. BCF (1)-(2) is figured convergent.

Theorem 2. Divergence of the series

$$
\sum_{n=1}^{\infty} \prod_{k=1}^{n}\left(a_{k, k}\right)^{(-1)^{n-k+1}}, \quad \sum_{n=1}^{\infty} \prod_{k=1}^{n}\left(a_{k+i, k}\right)^{(-1)^{n-k+1}}, \quad \sum_{n=1}^{\infty} \prod_{k=1}^{n}\left(a_{k, k+j}\right)^{(-1)^{n-k+1}}
$$

$i, j=1,2, \ldots$, is necessary condition of figured convergence of BCF (1)-(2) whose elements satisfy conditions (12) and (16).

Proof. Using well known results of analytic theory of continued fractions $[8,11]$, we conclude that continued fraction (2) converges if and only if the series $\sum_{n=1}^{\infty} \prod_{k=1}^{n}\left(a_{k+i, k+j}\right)^{(-1)^{n-k+1}}$ diverges. It was shown above that every summand which appears in expressions for $\tilde{f}_{4 m+2 p+l}-\tilde{f}_{4 m}$, $m, l=0,1, \ldots, p=1,2, \ldots$, is positive under conditions (12), (16). If series $\sum_{n=1}^{\infty} \prod_{k=1}^{n}\left(a_{k, k}\right)^{(-1)^{n-k+1}}$ converges, then $\lim _{m \rightarrow \infty}\left(\tilde{f}_{4 m+2}-\tilde{f}_{4 m}\right) \geq \lim _{m \rightarrow \infty}\left(F_{0,0}^{(2 m+1)}-F_{0,0}^{(2 m)}\right)>0$, i.e. BCF (1)-(2) diverges. Let there exists such $i$ that series $\sum_{n=1}^{\infty} \prod_{k=1}^{n}\left(a_{k+i, k}\right)^{(-1)^{n-k+1}}$ converges. Then taking into account inequalities (19), (20), we obtain

$$
\begin{aligned}
& \tilde{f}_{4 m+2}-\tilde{f}_{4 m}>\frac{\left|F_{i, 0}^{\left(2 m+1-\left[\frac{i+1}{2}\right]\right)}-F_{i, 0}^{\left(2 m-\left[\frac{i+1}{2}\right]\right)}\right| \prod_{j=1}^{i}\left|a_{j, 0}\right|}{\prod_{j=1}^{i} \tilde{Q}_{j, 0}^{(4 m+2-j)} \tilde{Q}_{j, 0}^{(4 m-j)}} \\
& =\frac{\left|F_{i, 0}^{\left(2 m+1-\left[\frac{i+1}{2}\right]\right)}-F_{i, 0}^{\left(2 m-\left[\frac{i+1}{2}\right]\right) \mid}\right| \prod_{j=1}^{i}\left|a_{j, 0}\right|}{\prod_{j=1}^{\left[\frac{i+1}{2}\right]} \tilde{Q}_{2 j-1,0}^{(4 m-2 j+3)} \tilde{Q}_{2 j-1,0}^{(4 m-2 j+1)} \prod_{j=1}^{\left[\frac{i}{2}\right]} \tilde{Q}_{2 j, 0}^{(4 m-2 j+2)} \tilde{Q}_{2 j, 0}^{(4 m-2 j)}}, 4 m \geq i,
\end{aligned}
$$




$$
\lim _{m \rightarrow \infty}\left(\tilde{f}_{4 m+2}-\tilde{f}_{4 m}\right) \geq \frac{\prod_{j=1}^{i}\left|a_{j, 0}\right| \lim _{m \rightarrow \infty} \mid F_{i, 0}^{\left(2 m+1-\left[\frac{i+1}{2}\right]\right)}-F_{i, 0}^{\left(2 m-\left[\frac{i+1}{2}\right]\right) \mid}}{\prod_{j=1}^{\left.\frac{i+1}{2}\right]}\left(1+a_{2 j, 1}\right)^{2} \prod_{j=1}^{\left[\frac{i}{2}\right]}\left(1+a_{2 j+1,1}+\frac{a_{2 j+1,0}}{1-\left|a_{2 j+2,0}\right|}\right)^{2}}>0 .
$$

From assumption about convergence of the series $\sum_{n=1}^{\infty} \prod_{k=1}^{n}\left(a_{k, k+i}\right)^{(-1)^{n-k+1}}$ for some value $i$ and from inequalities (21), (22) it follows that

$$
\lim _{m \rightarrow \infty}\left(\tilde{f}_{4 m+2}-\tilde{f}_{4 m}\right) \geq \frac{\prod_{j=1}^{i}\left|a_{0, j}\right| \lim _{m \rightarrow \infty}\left|F_{0, i}^{\left(2 m+1-\left[\frac{i+1}{2}\right]\right)}-F_{0, i}^{\left(2 m-\left[\frac{i+1}{2}\right]\right)}\right|}{\prod_{j=1}^{\left[\frac{i+1}{2}\right]}\left(1+a_{1,2 j}\right)^{2} \prod_{j=1}^{\left[\frac{i}{2}\right]}\left(1+a_{1,2 j+1}+\frac{a_{0,2 j+1}}{1-\left|a_{0,2 j+2}\right|}\right)^{2}}>0 .
$$

Remark. "Fork property" for ordinary approximants of even order is not valid. Really, let $b_{0}=1, a_{i, k}=1, a_{2 k-1,0}=a_{0,2 k-1}=1, a_{2 k, 0}=a_{0,2 k}=-\frac{1}{2}, i, k=1,2, \ldots$ Then $f_{0}=1, f_{2}=2 \frac{5}{6}$, $f_{4}=2 \frac{139}{140}>f_{2}$.

Theorem 3. If sequence $\left\{\tilde{f}_{k}\right\}$ of figured approximants of BCF (1)-(2) whose elements satisfy conditions (12) and (16) converges, then sequece $\left\{f_{k}\right\}$ of ordinary approximants converges to the same limit.

Proof. Using the formulas (6)-(7), conditions (12) and (16) the following inequalities can be proved in much the same way as inequalities (19)-(20)

$$
\begin{aligned}
1 \leq & Q_{2 k, 0}^{(p)} \leq 1+a_{2 k+1,1}+\frac{a_{2 k+1,0}}{1-\left|a_{2 k+2,0}\right|}, \quad k=1,2, \ldots, \quad p=0,1, \ldots, \\
& 1-\left|a_{2 k, 0}\right| \leq Q_{2 k-1,0}^{(p)} \leq 1+a_{2 k, 1}, \quad k=1,2, \ldots, \quad p=0,1, \ldots, \\
1 \leq & Q_{0,2 k}^{(p)} \leq 1+a_{1,2 k+1}+\frac{a_{0,2 k+1}}{1-\left|a_{0,2 k+2}\right|}, \quad k=1,2, \ldots, \quad p=0,1, \ldots, \\
& 1-\left|a_{0,2 k}\right| \leq Q_{0,2 k-1}^{(p)} \leq 1+a_{1,2 k}, \quad k=1,2, \ldots, \quad p=0,1, \ldots .
\end{aligned}
$$

Using the formula (11), we consider the following differences $f_{4 m+p}-\tilde{f}_{4 m}, f_{4 n+p-2}-\tilde{f}_{4 n-2}$, $m, n, p=1,2, \ldots$. We set $Z_{n, 0}^{(1)}=0, Z_{n, 0}^{(2)}=0$,

$$
\begin{aligned}
& Z_{n, m}^{(1)}=\sum_{i=1}^{m} \frac{(-1)^{i}\left(F_{i, 0}^{(n-i)}-F_{i, 0}^{\left(\left[\frac{m-i}{2}\right]\right)}\right) \prod_{j=1}^{i} a_{j, 0}}{\prod_{j=1}^{i} Q_{j, 0}^{(n-j)} \tilde{Q}_{j, 0}^{(m-j)}}, m=1,2, \ldots, \quad n>m, \\
& Z_{n, m}^{(2)}=\sum_{i=1}^{m} \frac{(-1)^{i}\left(F_{0, i}^{(n-i)}-F_{0, i}^{\left(\left[\frac{m-i}{2}\right]\right)}\right) \prod_{j=1}^{i} a_{0, j}}{\prod_{j=1}^{i} Q_{0, j}^{(n-j)} \tilde{Q}_{0, j}^{(m-j)}}, m=1,2, \ldots, \quad n>m .
\end{aligned}
$$


Then

$$
\begin{aligned}
f_{4 m+p}-\tilde{f}_{4 m} & =F_{0,0}^{(4 m+p)}-F_{0,0}^{(2 m)}+Z_{4 m+p, 4 m}^{(1)}+Z_{4 m+p, 4 m}^{(2)} \\
& +\frac{\prod_{j=1}^{4 m+1} a_{j, 0}}{\prod_{j=1}^{4 m+1} \tilde{Q}_{j, 0}^{(4 m+p-j)} \prod_{j=1}^{4 m} \tilde{Q}_{j, 0}^{(4 m-j)}}+\frac{\prod_{j=1}^{4 m+1} a_{0, j}}{\prod_{j=1}^{4 m+1} Q_{0, j}^{(4 m+p-j)} \prod_{j=1}^{4 m} \tilde{Q}_{0, j}^{(4 m-j)}}
\end{aligned}
$$

From (23), (27)-(30) it follows that

$$
\begin{aligned}
& Z_{4 m+p, 4 m}^{(1)}=-\sum_{i=1}^{m} \frac{\left(F_{4 i-3,0}^{(4 m+p-4 i+3)}-F_{4 i-3,0}^{(2 m-2 i+1)}\right) \prod_{j=1}^{4 i-3}\left|a_{j, 0}\right|}{\prod_{j=1}^{4 i-3} Q_{j, 0}^{(4 m+p-j)} \tilde{Q}_{j, 0}^{(4 m-j)}} \\
& -\sum_{i=1}^{m} \frac{\left(F_{4 i-2,0}^{(4 m+p-4 i+2)}-F_{4 i-2,0}^{(2 m-2 i+1)}\right) \prod_{j=1}^{4 i-2}\left|a_{j, 0}\right|}{\prod_{j=1}^{4 i-2} Q_{j, 0}^{(4 m+p-j)} \tilde{Q}_{j, 0}^{(4 m-j)}}+\sum_{i=1}^{m} \frac{\left(F_{4 i-1,0}^{(4 m+p-4 i+1)}-F_{4 i-1,0}^{(2 m-2 i)}\right) \prod_{j=1}^{4 i-1}\left|a_{j, 0}\right|}{\prod_{j=1}^{4 i-1} Q_{j, 0}^{(4 m+p-j)} \tilde{Q}_{j, 0}^{(4 m-j)}} \\
& +\sum_{i=1}^{m} \frac{\left(F_{4 i, 0}^{(4 m-p-4 i)}-F_{4 i, 0}^{(2 m-2 i)}\right) \prod_{j=1}^{4 i}\left|a_{j, 0}\right|}{\prod_{j=1}^{4 i} Q_{j, 0}^{(4 m+p-j)} \tilde{Q}_{j, 0}^{(4 m-j)}} \\
& =\sum_{i=1}^{m} \frac{\left|F_{4 i-3,0}^{(4 m+p-4 i+3)}-F_{4 i-3,0}^{(2 m-2 i+1)}\right| \prod_{j=1}^{4 i-3}\left|a_{j, 0}\right|}{\prod_{j=1}^{4 i-3} Q_{j, 0}^{(4 m+p-j)} \tilde{Q}_{j, 0}^{(4 m-j)}}+\sum_{i=1}^{m} \frac{\left|F_{4 i-2,0}^{(4 m+p-4 i+2)}-F_{4 i-2,0}^{(2 m-2 i+1)}\right| \prod_{j=1}^{4 i-2}\left|a_{j, 0}\right|}{\prod_{j=1}^{4 i-2} Q_{j, 0}^{(4 m+p-j)} \tilde{Q}_{j, 0}^{(4 m-j)}} \\
& +\sum_{i=1}^{m} \frac{\left|F_{4 i-1,0}^{(4 m+p-4 i+2)}-F_{4 i-1,0}^{(2 m-2 i)}\right| \prod_{j=1}^{4 i-1}\left|a_{j, 0}\right|}{\prod_{j=1}^{4 i-1} \tilde{Q}_{j, 0}^{(4 m+p-j)} \tilde{Q}_{j, 0}^{(4 m-j)}}+\sum_{i=1}^{m} \frac{\left|F_{4 i, 0}^{(4 m+p-4 i)}-F_{4 i, 0}^{(2 m-2 i)}\right| \prod_{j=1}^{4 i}\left|a_{j, 0}\right|}{\prod_{j=1}^{4 i} Q_{j, 0}^{(4 m+p-j)} \tilde{Q}_{j, 0}^{(4 m-j)}}>0 .
\end{aligned}
$$

Similarly $Z_{4 m+p, 4 m}^{(2)}>0$. Consequently

$$
f_{4 m+p}-\tilde{f}_{4 m}>0, \quad m, l=0,1, \ldots, \quad p=1,2, \ldots
$$

Further,

$$
\begin{aligned}
f_{4 n+p-2}-\tilde{f}_{4 n-2} & =F_{0,0}^{(4 n+p-2)}-F_{0,0}^{(2 n-1)}+Z_{4 n+p-2,4 n-2}^{(1)}+Z_{4 n+p-2,4 n-2}^{(2)} \\
& +\frac{\prod_{j=1}^{4 n-1} a_{j, 0}}{\prod_{j=1}^{4 n-1} Q_{j, 0}^{(4 n+p-2-j)} \prod_{j=1}^{4 n-2} \tilde{Q}_{j, 0}^{(4 n-2-j)}}+\frac{\prod_{j=1}^{4 n-1} a_{0, j}}{\prod_{j=1}^{4 n-1} Q_{0, j}^{(4 n+p-2-j)} \prod_{j=1}^{4 n-2} \tilde{Q}_{0, j}^{(4 n-2-j)}} .
\end{aligned}
$$


From (25), (27)-(30) it follows that

$$
\begin{aligned}
& Z_{4 n+p-2,4 n-2}^{(1)}=-\sum_{i=1}^{n} \frac{\left(F_{4 i-3,0}^{(4 n+p-4 i+1)}-F_{4 i-3,0}^{(2 n-2 i)}\right) \prod_{j=1}^{4 i-3}\left|a_{j, 0}\right|}{\prod_{j=1}^{4 i-3} Q_{j, 0}^{(4 n+p-2-j)} \tilde{Q}_{j, 0}^{(4 n-2-j)}}-\sum_{i=1}^{n} \frac{\left(F_{4 i-2,0}^{(4 n+p-4 i)}-F_{4 i-2,0}^{(2 n-2 i)}\right) \prod_{j=1}^{4 i-2}\left|a_{j, 0}\right|}{\prod_{j=1}^{4 i-2} Q_{j, 0}^{(4 n+2 p+l-2-j)} \tilde{Q}_{j, 0}^{(4 n-2-j)}} \\
& +\sum_{i=1}^{n-1} \frac{\left(F_{4 i-1,0}^{(4 n+p-4 i-1)}-F_{4 i-1,0}^{(2 n-2 i-1)}\right) \prod_{j=1}^{4 i-1}\left|a_{j, 0}\right|}{\prod_{j=1}^{4 i-1} Q_{j, 0}^{(4 n+p-2-j)} \tilde{Q}_{j, 0}^{(4 n-2-j)}}+\sum_{i=1}^{n-1} \frac{\left(F_{4 i, 0}^{(4 n+p-4 i-2)}-F_{4 i, 0}^{(2 n-2 i-1)}\right) \prod_{j=1}^{4 i}\left|a_{j, 0}\right|}{\prod_{j=1}^{4 i} Q_{j, 0}^{(4 n+p-2-j)} \tilde{Q}_{j, 0}^{(4 n-2-j)}}<0 .
\end{aligned}
$$

Similarly $Z_{4 n+p-2,4 n-2}^{(2)}<0$. Consequently

$$
f_{4 n+p-2}-\tilde{f}_{4 n-2}<0, \quad n, p=1,2, \ldots
$$

Taking into account the inequalities (17), (31), (32) we obtain $\tilde{f}_{4 m}<f_{4 m+p+2}<\tilde{f}_{4 m+2}$, $m=0,1, \ldots, p=1,2, \ldots$. In the case of convergence of sequence $\left\{\tilde{f}_{2 k}\right\}$ we conclude that $\lim _{n \rightarrow \infty} \tilde{f}_{n}=\lim _{k \rightarrow \infty} f_{k}$.

\section{REFERENCES}

[1] Antonova T.M., Vozna S.M. On one convergence criterion of branched continued fraction of the special form with real elements. Prykl. Probl. Mekh. Mat. 2016, 14, 16-24. (in Ukrainian)

[2] Antonova T.M., Vozna S.M. Some properties of approximants for branched continued fractions of the special form with nonpositive partial numerators. Bukovyn Mat. Zh. 2017, 5 (1-2), 6-15. (in Ukrainian)

[3] Antonova T.M., Sus' O.M. On some sequences of the sets of uniform convergence for two-dimensional continued fractions. J. Math. Sci. (N.Y.) 2017, 222 (1), 56-69. doi: 10.1007/s10958-017-3282-y (translation of Mat. Metody Fiz.-Mekh. Polya 2015, 58 (1), 47-56. (in Ukrainian))

[4] Antonova T.M., Sus' O.M. Sufficient conditions for the equivalent convergence of sequences of different approximants for two-dimensional continued fractions. J. Math. Sci. (N.Y.) 2018, 228 (1), 1-10. doi: 10.1007/s10958-017-3601-3 (translation of Mat. Metody Fiz.-Mekh. Polya 2015, 58 (4), 7-14. (in Ukrainian))

[5] Bodnar D.I. Branched continued fractions. Nauk. dumka, Kyiv, 1986. (in Russian)

[6] Bodnar D.I., Bilanyk I.B. Convergence criterion for branched continued fraction of the special form with positive elements. Carpathian Math. Publ. 2017, 9 (1), 13-21. doi: 10.15330/cmp.9.1.13-21

[7] Dmytryshyn R.I. On the convergence criterion for branched continued fractions with independent variables. Carpathian Math. Publ. 2017, 9 (2), 120-127. doi: 10.15330/cmp.9.2.120-127

[8] Khovanskii A.N. The application of continued fractions and their generalizations to problems in approximation theory. P. Noordhoff, Groningen, 1963.

[9] Kuchmins'ka Kh.Yo. Corresponding and associated branched continued fractions for double power series. Dop. AN UkrSSR. Ser. A. 1978, 7, 614-618. (in Ukrainian)

[10] Kuchmins'ka Kh.Yo. Two-dimensional continued fractions. Ya. S. Pidstryhach Institute for Appl. Probl. of Mech. and Math. NAS of Ukraine, Lviv, 2010. (in Ukrainian)

[11] Lorentzen L., Waadeland H. Continued fractions with applications. In: Brezinski C., Wuytack L. (Eds.) Studies in Computational Mathematics, 3. Noth Holland, Amsterdam, 1992. 
[12] Murphy J., O'Donohoe M.R. A two-variable generalization of the Stieltjes-type continued fractions. J. Comput. Appl. Math. 1978, 4(3), 181-190. doi: 10.1016/0771-050x(78)90002-5

[13] Siemaszko W. Branched continued fractions for double power series. J. Comput. Appl. Math. 1980, 6 (2), 121-125. doi: 10.1016/0771-050x(80)90005-4

Received 23.02.2018

Revised 12.05.2018

Антонова Т.М., Амитришин М.В., Возна С.М. Аеякі властивості наближень гіллястих ланиюгових дробів спеціального вигляду з додатними і знакопочережними иастинними иисельниками // Карпатські матем. публ. — 2018. - Т.10, №1. — С. 3-13.

Стаття присвячена дослідженню збіжності одного із узагальнень ланцюгових дробів — гіллястих ланцюгових дробів спеціального вигляду з двома гілками розгалужень. Такі дроби, так само як і двовимірні неперервні дроби та гіллясті ланцюгові дроби з двома нерівнозначними змінними, пов'язані з проблемою відповідності між формальним подвійним степеневим рядом і послідовністю раціональних наближень функції двох змінних.

На відміну від неперервних дробів, наближення яких будуються однозначно, існує багато способів побудови наближень гіллястих ланцюгових дробів загального та спеціального вигляду. У роботі розглянуто звичайні наближення та одну з конструкцій фігурних наближень досліджуваних гіллястих ланцюгових дробів, яка пов' язана із задачею відповідності.

Розглянуто деякі властивості наближень таких гіллястих ланщюгових дробів спеціального вигляду, частинні чисельники яких додатні і знакопочережні, а частинні знаменники дорівнюють одиниці. Встановлено деякі необхідні і достатні умови фігурної збіжності. Аоведено, що за сформульованих умов із збіжності послідовності фігурних наближень випливає збіжність послідовності звичайних наближень до тої самої границі.

Ключові слова і фрази: гіллястий ланцюговий дріб спеціального вигляду, звичайні наближення, фігурні наближення, збіжність, фігурна збіжність. 